\title{
RPA quasi-elastic responses in infinite and finite nuclear systems
}

\author{
Eduardo Bauer ${ }^{1,3}$ and Giampaolo Co, ${ }^{2}$ \\ ${ }^{1}$ Departamento de Física, Facultad de Ciencias Exactas, Universidad Nacional de La Plata, \\ La Plata, 1900, Argentina \\ 2 Dipartimento di Fisica, Università di Lecce, and Istituto Nazionale di Fisica Nucleare, \\ sezione di Lecce, I-73100 Lecce, Italy
}

Received 17 April 2001

Published 17 July 2001

Online at stacks.iop.org/JPhysG/27/1813

\begin{abstract}
Quasi-elastic responses in nuclear matter and in ${ }^{12} \mathrm{C}$ and ${ }^{40} \mathrm{Ca}$ nuclei are calculated with the ring approximation to investigate the finite-size effects on the electromagnetic quasi-elastic responses. A method to simulate these effects in infinite-systems calculations is proposed. The sensitivity of the results to the various terms of the residual interaction is studied. The results of nuclear matter random-phase approximation calculations are compared with those obtained in the ring approximation to demonstrate the importance of the exchange terms.
\end{abstract}

\section{Introduction}

The quasi-elastic excitation of the nucleus is particularly interesting for nuclear structure studies because of the interplay between single-particle and many-body effects. The excitation energies characterizing the quasi-elastic peak are well above the nucleon emission threshold, therefore, one or more nucleons are ejected from the nucleus. Before being ejected, any nucleon can interact with the other nucleons composing the nucleus. Considering this effect in a finite-nucleus formalism is a difficult task because the nuclear final states are described in terms of their total angular momentum and the presence of the continuum implies the sum on a large number of possible configurations.

In a nuclear matter formalism one takes advantage of the translational invariance to simplify the description of the final state of the hadronic system. The use of the infinitesystem formalism is rather appropriate for the quasi-elastic electron excitation, since excitation energies and transferred momenta are such that the excitation process is well localized within the nucleus and collective surface excitations are negligible. In any case additional approximations (say, a variable Fermi momentum or the local density approximation) have been considered in the literature to simulate finite-size effects.

3 Fellow of the Consejo Nacional de Investigaciones Científicas y Técnicas, CONICET. 
We can classify the necessary ingredients to describe the process into three main issues: the single-particle basis, the inclusion of initial- and/or final-state interactions and the operator describing the action of the external probe. Regarding the first point we have already mentioned that some work treats the nucleus as a finite system [1-11], while others use a nuclear matter approach [12-25].

Referring to the second point, one of the simplest approaches to include initial- and final-state interactions is the random-phase approximation (RPA), with or without exchange terms (the last approach is called the ring approximation). In the RPA [1-5, 12-14] oneparticle-one-hole $(1 \mathrm{p} 1 \mathrm{~h})$ excitations are summed up to infinite order. One step up in complexity is the so-called second RPA (SRPA) [6,15] which, in addition to the 1p1h excitations, also considers those generated by 2p2h. The extended RPA (ERPA) [7, 16, 17]; contains ground-state correlations beyond RPA. The Green function approach of [8] is based on a philosophy similar to that of the SRPA. In this approach the relationship between forward virtual Compton scattering and inclusive electron scattering is used to construct a one-body approximation to quasi-elastic electron scattering. The role of the short-range correlations has been investigated within the framework of the correlated basis function theory $[10,18]$.

Concerning the third point, the external operator is usually represented by electromagnetic one-body operators, but in the transverse channel it is important to include two-body terms as the meson exchange currents (MEC) $[9,11,23,25]$, and the excitation of the virtual or real $\Delta(1232)$ resonance [19-22].

In the present paper we investigate the sources of some inconsistencies between finiteand infinite-nucleus calculations. For example, it seems that once the residual interactions have been fixed, the effects of the ring approximation are larger in nuclear matter than in finite nuclei.

To simulate the finite-size effects in the quasi-elastic peak, we have performed infinitesystems calculations with a diffused Fermi surface. Then, we have studied the effects on the responses of the various characteristics of the interaction, such as the different channels, the range and the density dependence. The infinite-systems results have been compared with the calculations performed for the ${ }^{12} \mathrm{C}$ and ${ }^{40} \mathrm{Ca}$ nuclei, two doubly magic nuclei with the same number of protons and neutrons. The agreement we have finally obtained between the two kinds of calculations is satisfactory, even for the nucleus ${ }^{12} \mathrm{C}$, which is supposed to be relatively light to be well represented as an infinite system of nucleons.

Encouraged by this result, we have evaluated the response functions within the nuclear matter RPA framework of [13] to investigate the effects of the exchange terms neglected in the ring approximation.

The paper is organized as follows. In section 2 we briefly present the effective theories used to perform the calculations. In section 3 we compare the results obtained in finite nuclei and nuclear matter both for the free and the ring responses, and we present the nuclear matter RPA responses. Finally, in section 4 we draw our conclusions.

\section{Formalism}

The response function for inclusive quasi-elastic electron scattering is given by

$$
R(\boldsymbol{q}, \omega)=-\frac{1}{\pi} \operatorname{Im}\left\langle 0\left|\mathcal{O}^{\dagger}(\boldsymbol{q}) G(\omega) \mathcal{O}(\boldsymbol{q})\right| 0\right\rangle
$$


where $\omega$ represents the excitation energy and $q$ the 3-momentum transferred by the electron. The nuclear ground state is denoted as $|0\rangle$, while $\mathcal{O}(\boldsymbol{q})$ is the excitation operator and $G(\omega)$ the polarization propagator,

$$
G(\omega)=\frac{1}{\omega-H+\mathrm{i} \eta}-\frac{1}{\omega+H+\mathrm{i} \eta}
$$

where $H$ is the nuclear Hamiltonian. We introduce the operator $P$, which projects onto $1 \mathrm{p} 1 \mathrm{~h}$ configurations. The method of building these configurations depends on the particular choice of the single-particle basis. In nuclear matter the single-particle wavefunctions are plane waves, while in a finite nucleus they are eigenfunctions of the one-body Schrödinger equation for a mean-field potential. In our case we use a real, spherical, Woods-Saxon potential.

The response function generated by $1 \mathrm{p} 1 \mathrm{~h}$ excitations can be expressed as

$$
R_{P P}(\boldsymbol{q}, \omega)=-\frac{1}{\pi} \operatorname{Im}\left\langle 0\left|\mathcal{O}^{\dagger}(\boldsymbol{q}) P G(\omega) P \mathcal{O}(\boldsymbol{q})\right| 0\right\rangle .
$$

The evaluation of $R_{P P}$ is not straightforward since the nuclear Hamiltonian is in general not diagonal in the $1 \mathrm{p} 1 \mathrm{~h}$ basis. The solution of the problem is the RPA response. In this paper, we shall compare finite-nucleus results obtained within the ring approximation of [5], with the corresponding nuclear matter responses.

In most calculations performed in infinite systems the Fermi surface which separates, in momentum or energy space, the hole from the particle states, is a sharp step function. In the next section, we shall show that this approximation reproduces rather well the position and the shape of the free responses calculated in finite nuclei. However, the ring responses are appreciably different when evaluated in nuclear matter and in finite nuclei. The original motivation of this paper was to explain this discrepancy. The first attempt to improve our nuclear matter model, is to replace the step function representing the momentum distribution of particles and holes, by a more realistic one. For the holes with momentum $\boldsymbol{h}$ we make the substitution,

$$
\theta\left(k_{F}-|\boldsymbol{h}|\right) \rightarrow n(h)
$$

where $k_{F}$ is the Fermi momentum, $h \equiv|\boldsymbol{h}|$, and

$$
n(h)=\frac{1}{1+\mathrm{e}^{\left(h-k_{F}\right) / a}}
$$

with $a$ being a constant to be adjusted. An analogous expression can be obtained for particles.

In the next section we shall compare results obtained with this smoothed Fermi surface in the ring approximation with those obtained in finite-systems calculations. With the same smoothed momentum distribution we have performed RPA calculations following the computational scheme developed in [13] which we describe briefly. In nuclear matter, direct RPA terms can be summed up to infinite order (ring series), but, in general, it is not possible to find a closed form to sum all the exchange terms. Normally these last terms are considered perturbatively and, for numerical reasons, this is done up to the second order. On the other hand, it is possible to make the full summation of the exchange terms when a contact interaction is used. Full sums are also possible for separable interactions. To exploit this feature we rewrite the residual interaction $V$, as

$$
V=V_{\text {contact }}+\tilde{V}
$$

The $V_{\text {contact }}$ term is a contact interaction conveniently chosen to make $\tilde{V}$ small. For $V_{\text {contact }}$ both direct and exchange terms are summed up to infinite order while $\tilde{V}$ is perturbatively considered up to the second order, which we found to account reasonably well for the whole 
sum. Interference terms between $V_{\text {contact }}$ and $\tilde{V}$ are included up to infinite order in $V_{\text {contact }}$ and up to second order in $\tilde{V}$. A more detailed description of the method can be found in [13].

The finite-nucleus calculations have been done within the Fourier-Bessel computational scheme adopted in [5]. The single-particle basis is generated by using a Woods-Saxon mean field whose parameters have been fixed to reproduce experimental RMS radii and singleparticle energies of the bound states close to the Fermi surface. By neglecting the exchange diagrams, the RPA equations are rewritten in terms of local density functions which are expanded on a Fourier-Bessel basis. In this manner the problem to be solved, for every value of the excitation energy, is the diagonalization of a matrix whose dimensions are four times the number of the Fourier-Bessel expansion coefficients. More details concerning this method can be found in [24].
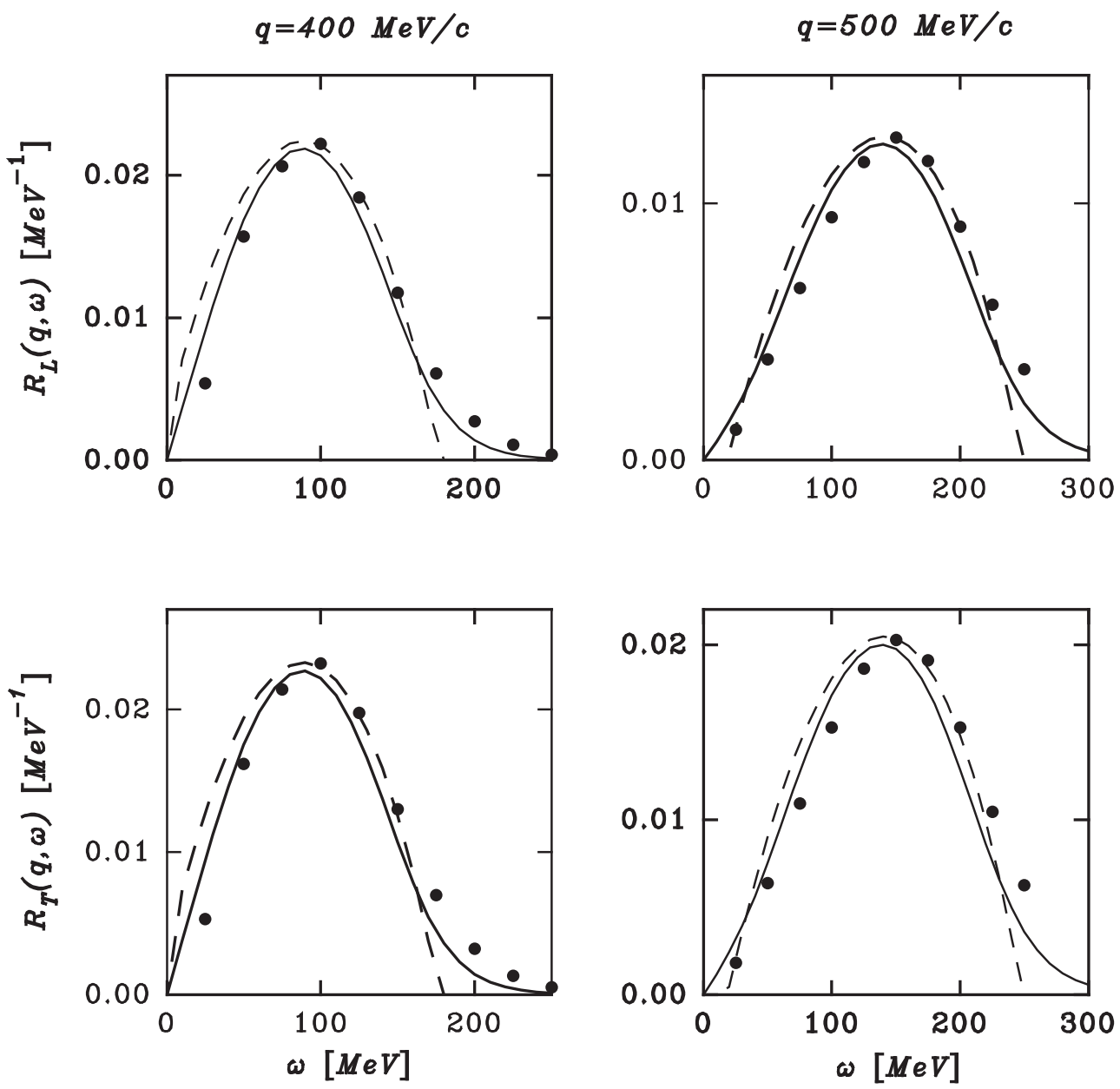

Figure 1. Nuclear-matter-free responses for two values of the momentum transfer compared with the continuum shell model ${ }^{12} \mathrm{C}$ responses (full circles). The full curves have been obtained using a diffused Fermi surface, the broken ones with a step functions Fermi surface. In both cases the values of the parameters have been modified to reproduce the finite-nucleus responses. 


\section{Results}

In this section we compare finite-nucleus results with those of nuclear matter. This is done for two nuclei: ${ }^{12} \mathrm{C}$ and ${ }^{40} \mathrm{Ca}$. The ${ }^{12} \mathrm{C}$ nucleus is perhaps too light to be appropriately described in terms of nuclear matter. On the other hand, we wanted to also test our model in extreme situations and, last but not least, the finite-nucleus calculations are much less involved than in the ${ }^{40} \mathrm{Ca}$ case.

The transferred momenta analysed are $q=400 \mathrm{MeV} c^{-1}$ and $q=500 \mathrm{MeV} c^{-1}$. These values are sufficiently large to eliminate the presence of collective surface vibrations, and at the same time, sufficiently small to require a limited number of partial waves. In the finite-nucleus calculations we sum multipole excitations up to angular momentum $J=12$ [9].

The first step of our calculations consists in fixing the values of $k_{F}$ and $a$ in equation (5) to reproduce the finite nucleus-free responses. For ${ }^{12} \mathrm{C}$ we have obtained the values $k_{F}=0.85 \mathrm{fm}^{-1}$ and $a=0.20 \mathrm{fm}^{-1}$, and for ${ }^{40} \mathrm{Ca}, k_{F}=1.0 \mathrm{fm}^{-1}$ and
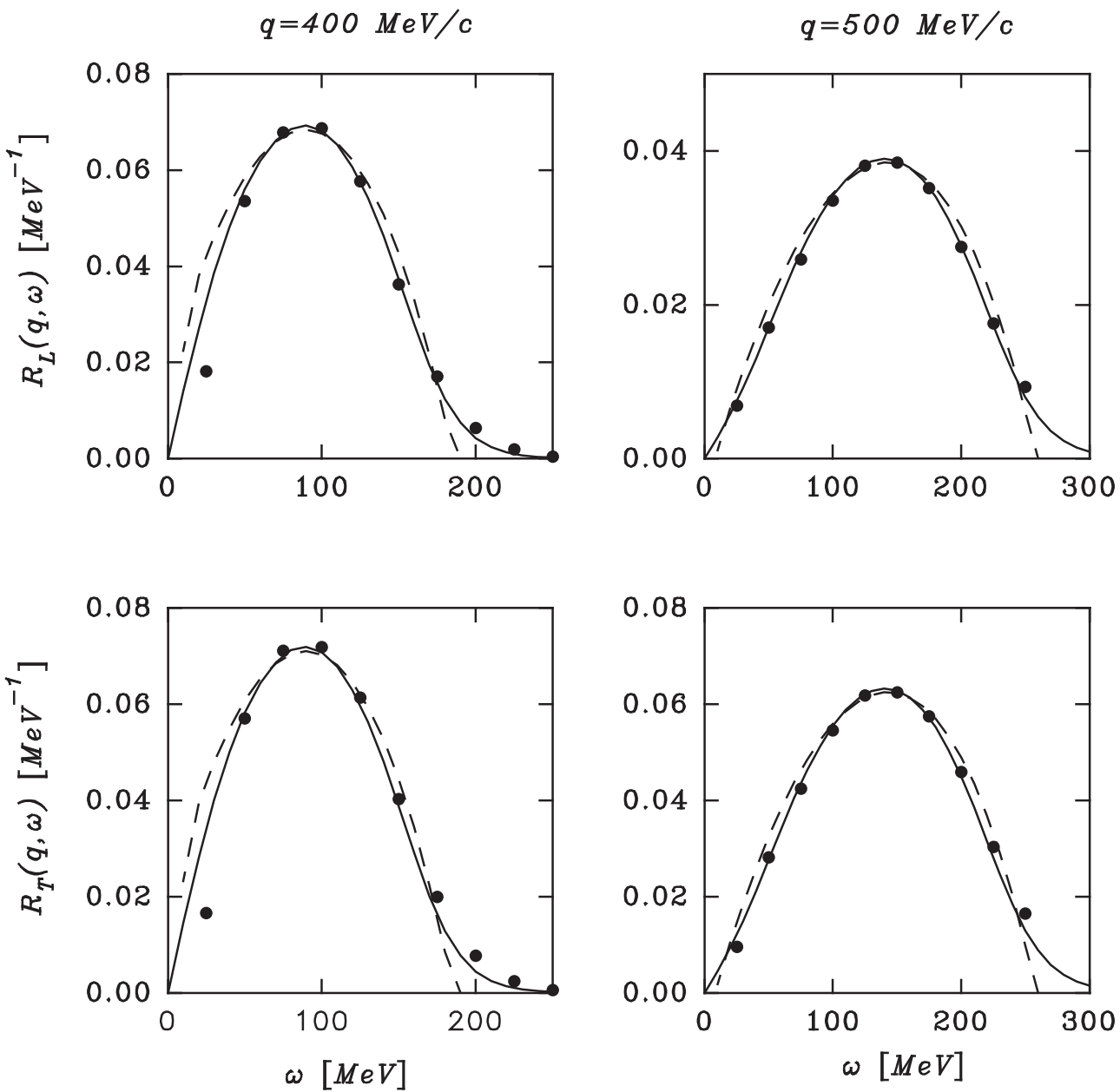

Figure 2. The same as in figure 1 but for ${ }^{40} \mathrm{Ca}$. 

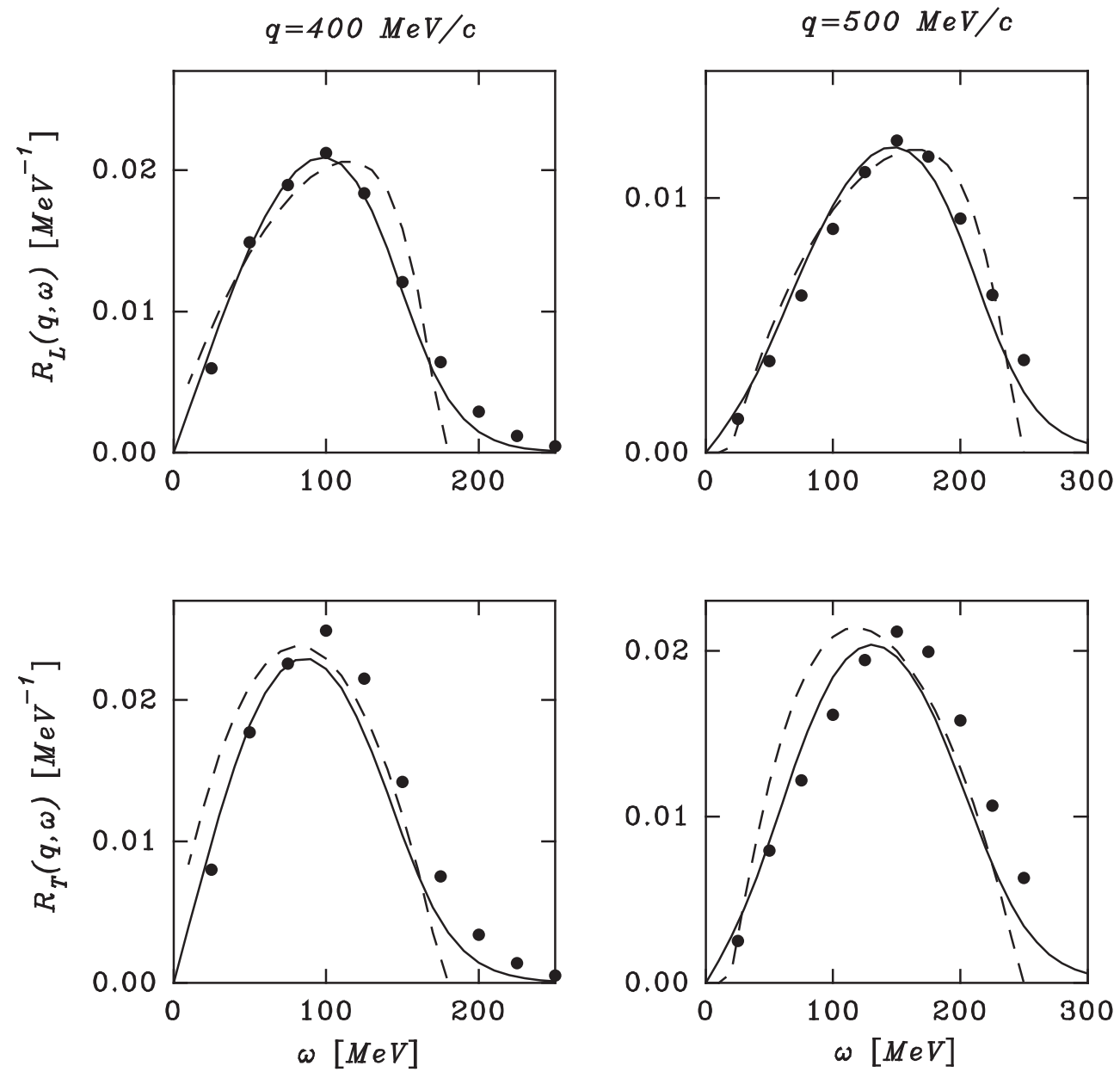

Figure 3. ${ }^{12} \mathrm{C}$ responses calculated in the ring approximation with the polarization potential. The meaning of the curves and of the symbols is the same as in figure 1.

$a=0.17 \mathrm{fm}^{-1}$. The comparison with the finite-nuclei responses is shown in figures 1 and 2, where we have also added the results obtained with a step-function Fermi surface represented by the broken curves. In this last case we have used the procedure of [11] to fix the value of the Fermi momentum, obtaining the values $k_{F}=1.09 \mathrm{fm}^{-1}$ for ${ }^{12} \mathrm{C}$ and $k_{F}=1.19 \mathrm{fm}^{-1}$ for ${ }^{40} \mathrm{Ca}$. These values are notably different from those fixed by the smoothed momentum distribution. As expected, $k_{F}$ increases with increasing mass number. We succeeded in obtaining a satisfactory agreement with the finite-nucleus responses, especially for ${ }^{40} \mathrm{Ca}$, in the case of a diffused Fermi surface. Our calculations done with a sharp Fermi surface do not produce the high-energy tail of the finite-nucleus response.

The comparison between the various responses calculated in the ring approximation is shown in figures 3 and 4 for the ${ }^{12} \mathrm{C}$ and ${ }^{40} \mathrm{Ca}$ nuclei, respectively. In these figures the meaning of the symbols is analogous to that of the previous figures. The interaction used in these 

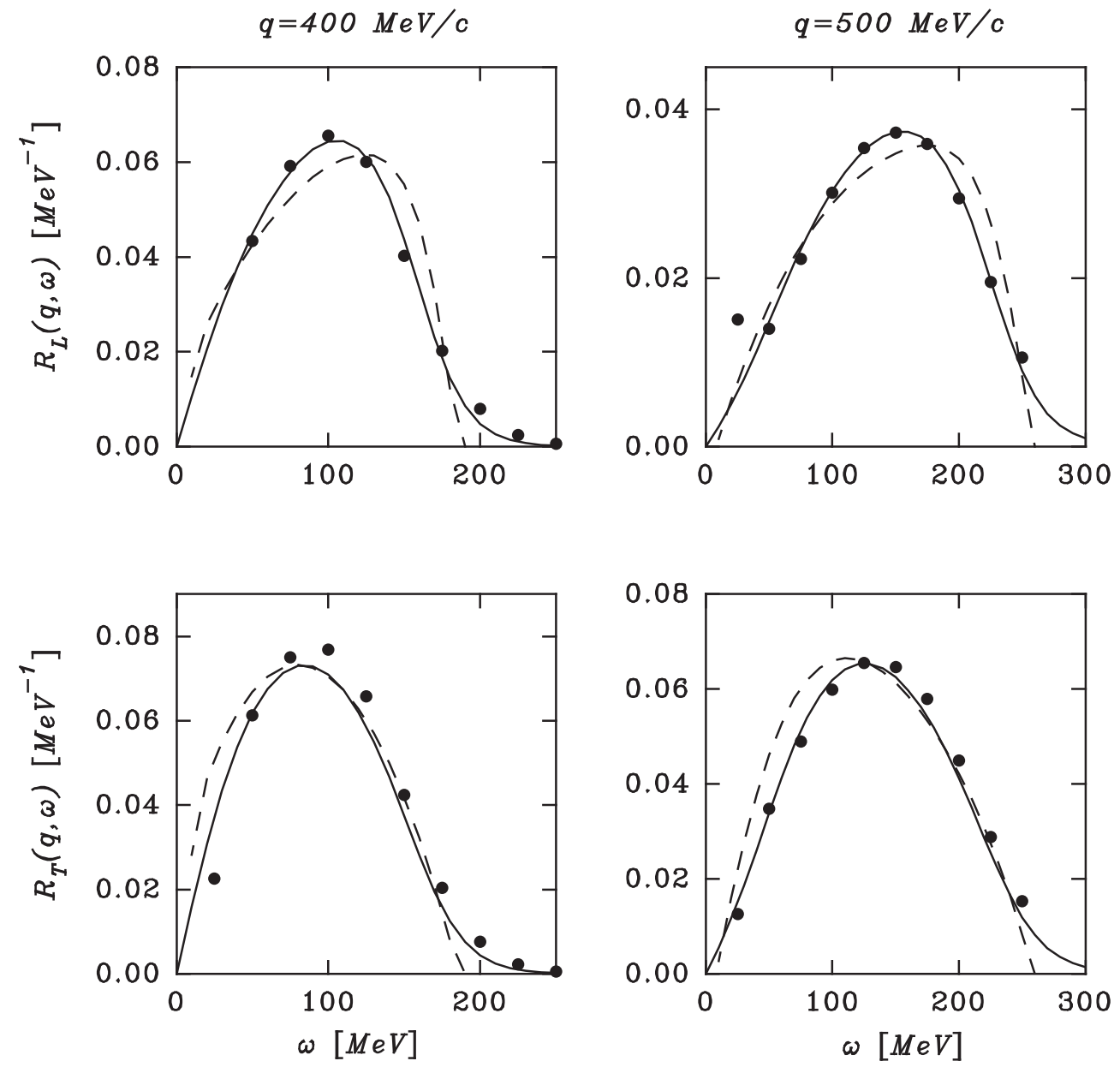

Figure 4. Same as in figure 3 for ${ }^{40} \mathrm{Ca}$.

calculations is the finite-range polarization potential also utilized in [5]. The nuclear matter calculations have been done with the values of $k_{F}$ and $a$ previously fixed.

Two observations should be made about these results. The first concerns the fact that, in general, the full curves reproduce the finite-nucleus results better than the broken ones. Secondly, we observe that the longitudinal responses are better reproduced than the transverse ones.

The first observation induces one to conclude that the sharp Fermi surface, even with an effective value of the Fermi momentum, is unable to reproduce the finite-nucleus results. This feature does not depend from the residual interaction, as we show in figure 5 where the various ${ }^{12} \mathrm{C}$ responses have been calculated, always in the ring approximation, with a zerorange Migdal interaction. We have used the following values of the parameters of this force: $f_{0}=386, f_{0}^{\prime}=289.5, g_{0}=106.2$ and $g_{0}^{\prime}=135.1$, expressed in units of $\mathrm{MeV} \mathrm{fm}^{3}$. These values have been chosen to magnify some of the effects we want to discuss. Specifically, the large value of $f_{0}$, ten times larger than that normally used [26], enhances the difference 

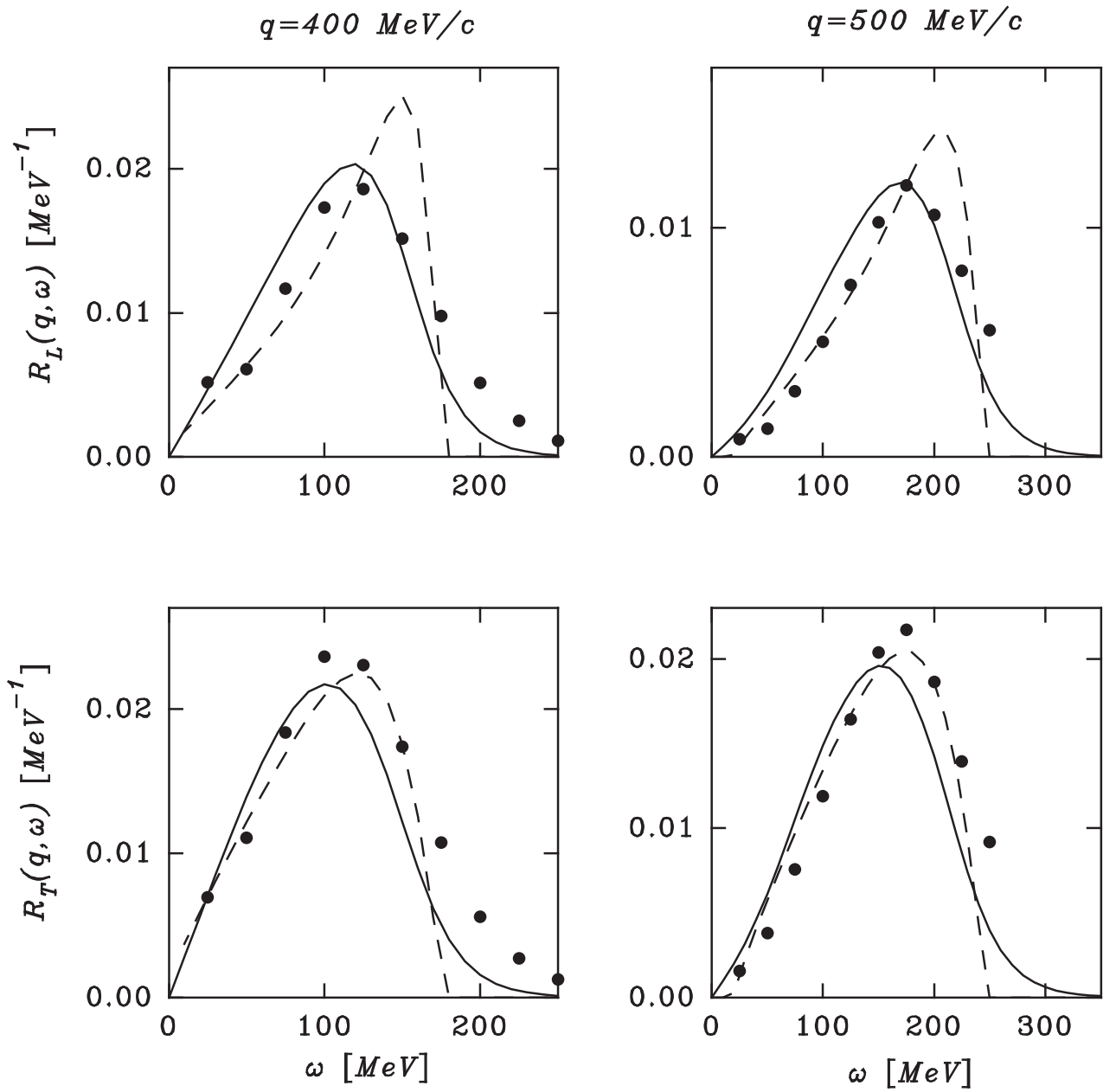

Figure 5. ${ }^{12} \mathrm{C}$ responses calculated in the ring approximation with the zero-range Migdal interaction.

between the nuclear matter calculations in the longitudinal response. Here the poor quality of the sharp Fermi surface calculations becomes more evident. Our diffused Fermi surface calculation is able to reproduce the finite-nucleus results rather well even in these extreme conditions.

Concerning the observation that, the longitudinal responses are always better reproduced than the transverse ones, we have verified that this fact is due to the differences between infinite and finite systems. The isospin channel of the force does not contribute in the ring approximation calculations of nuclear matter transverse responses, while it does in finitenuclei calculations. The effects of this difference become evident by comparing the transverse responses of figures 5 and 6 . In the latter figure the ${ }^{12} \mathrm{C}$ transverse responses have been calculated with the contact interaction described above but without the isospin channel of the force, i.e. by setting $f_{0}^{\prime}=0$. The agreement between the finite-nucleus responses and those obtained with the diffused Fermi surface is comparable with that obtained in the longitudinal case. 

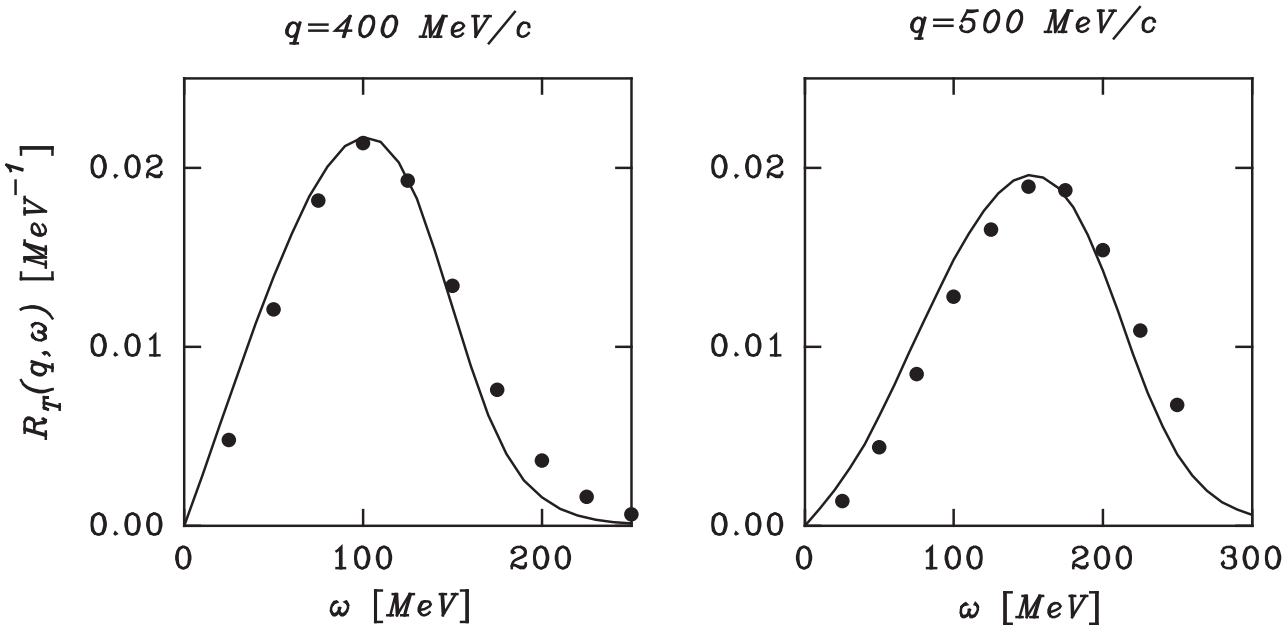

Figure 6. Transverse ${ }^{12} \mathrm{C}$ responses calculated in the ring approximation with a contact interaction. The spin-isospin term was left out.

We should note the fact that the polarization potential has a density dependence in the scalar and isospin channels. We have introduced this linear dependence of the force from the density by using internal and external parameters of the force,

$$
F(r)=F^{e x t}+\left(F^{i n t}-F^{e x t}\right) \frac{\rho(r)}{\rho(r=0)}
$$

Our nuclear matter calculations have been done by using the force parameters defined for the nuclear interior. A check of the sensitivity of the results to the density dependence of the force has been done by performing finite-nucleus calculations where the density dependence was switched off and the average values between internal and external parameters were used. The differences between the transverse responses with and without density dependence are within the numerical accuracy of the calculation. In the case of the longitudinal response the differences are not completely negligible, but they are of the same order of magnitude as those found between nuclear matter and finite-nuclei results.

In figures 7 and 8 we compare the RPA nuclear matter responses (full curves) with those evaluated in the ring approximation (broken curves). Both calculations have been done by using the polarization potential and the diffused Fermi surface. This comparison shows the effects of the exchange diagrams evaluated in RPA and neglected in the ring approximation. For the particular interaction used these effects are notable, especially in the longitudinal responses and for low values of the momentum transfer.

In the same figures we also present the free responses (dotted curves) and the ${ }^{12} \mathrm{C}$ and ${ }^{40} \mathrm{Ca}$ experimental points. Our results show that the major source of disagreement between free responses and data is produced by correlations beyond the RPA. In [5] the role of the final-state interactions and of the effective mass was pointed out. The inclusion of these two effects within a simplified model produces a good agreement with the ${ }^{40} \mathrm{Ca}$ data [9]. The same model is, however, unable to explain the ${ }^{12} \mathrm{C}$ transverse response data. 

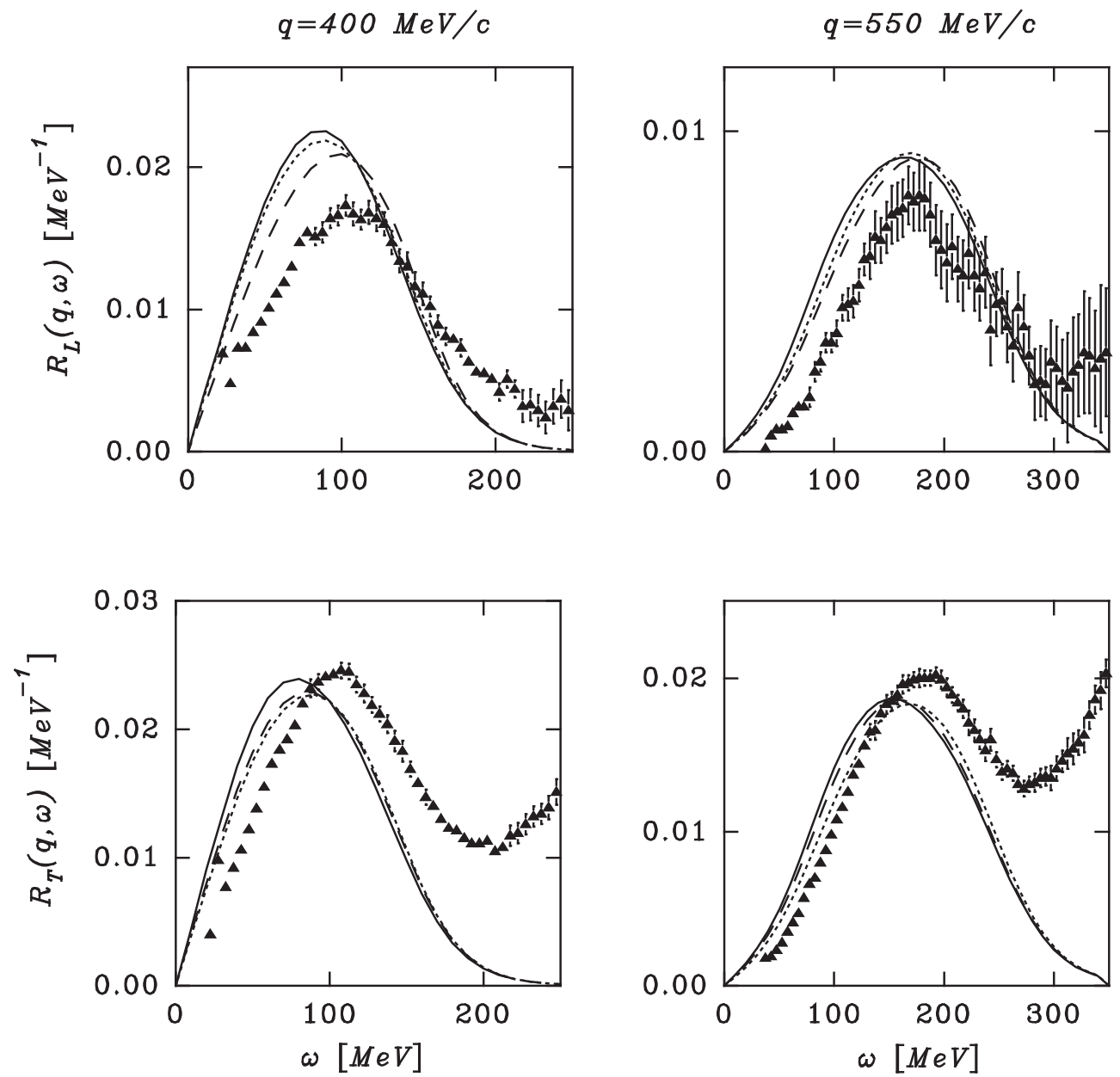

Figure 7. ${ }^{12} \mathrm{C}$ responses calculated in the RPA (full curves) and the ring approximation (broken curves) with the polarization potential. The dotted curves show the free responses. Data from [27].

\section{Conclusions}

In this paper we have investigated the differences between the ring approximation and the RPA calculations of electromagnetic quasi-elastic responses in nuclear matter and finite nuclei. We understand why, for a given residual interaction, the nuclear matter RPA effects found in the literature are larger than those produced in finite nuclear systems. This is mainly due to the use, in traditional nuclear matter calculations, of sharp Fermi surfaces in momentum space.

In our investigation we have adopted, for the nuclear matter calculations, a diffused momentum distribution of particles and holes. We have described this momentum distribution with a simple Fermi function depending on two parameters whose values have been adjusted to reproduce the finite nuclei-free responses.

This approach should be compared with the most commonly used method to account for finite-nucleus effects in nuclear matter calculations: the local density approximation. Unfortunately, this comparison is not straightforward. In LDA the responses are calculated 

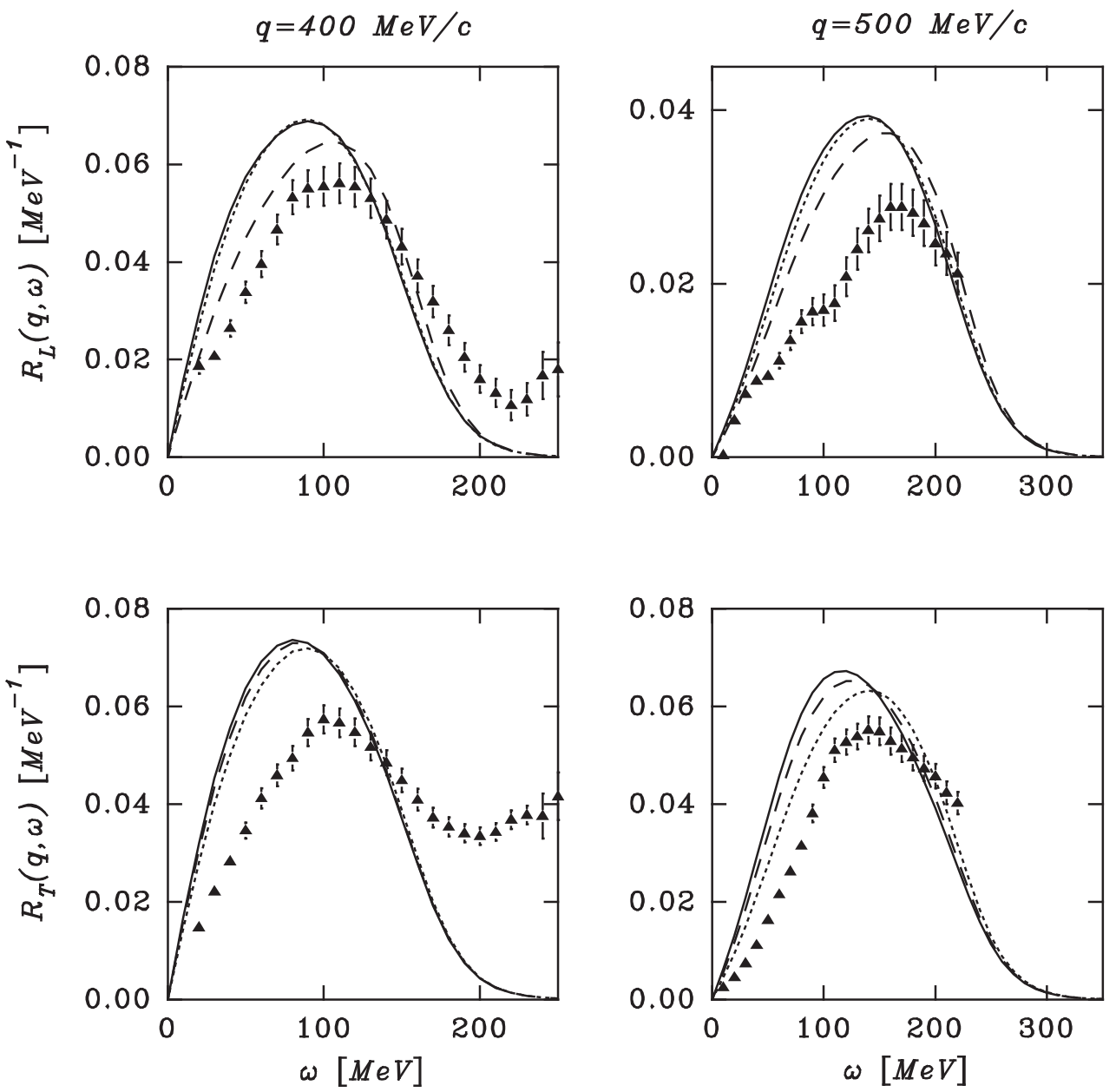

Figure 8. The same as in figure 7 but for ${ }^{40} \mathrm{Ca}$. Data from [28].

for several values of the Fermi momentum and then they are appropriately averaged to obtain the final result. In the averaging procedure, how the responses for each $k_{F}$ are weighted differs from nucleus to nucleus. However, for each $k_{F}$ a sharp Fermi surface is employed and this implies that the second term on the right-hand side of equation (2) does not contribute. These terms, however, give a contribution when a smooth Fermi surface is considered. This contribution is normally small but it becomes appreciable when $\omega \leqslant 20 \mathrm{MeV}$. This is the formal difference between our method and the LDA.

We used our smooth Fermi surface model to calculate the ${ }^{12} \mathrm{C}$ and ${ }^{40} \mathrm{Ca}$ responses for different values of the momentum transfer in the ring approximation. The agreement with the finite-nucleus results is excellent for the longitudinal responses. The small differences between the transverse responses are due to the isospin part of the residual interaction which is not active in nuclear matter calculations.

The smooth Fermi surface prescription has been used to make nuclear matter RPA calculations, i.e. calculations where the exchange diagrams are also considered. We also used the polarization potential in this case, but this creates a double-counting problem, since this 
interaction was constructed in [29] to consider, in an average manner, the contribution of the exchange diagrams. However, the aim of these RPA calculations was to test their feasibility. These kinds of calculations have been done in finite nuclei [1-4], but they require a large computational effort, while our method is simpler. For more realistic RPA calculations one has to choose a suitable residual interaction to avoid the double-counting problem.

The comparison with the experimental data shows that even nuclear matter ring or RPA calculations cannot explain the disagreement with the theory. Clearly, our results are related to the use of the polarization potential, whose qualities as an appropriate residual interaction can be widely discussed. On the other hand, all the finite-nuclei calculations we know, which use finite-range residual interactions, obtain results analogous to ours, showing small effects of RPA correlations on the free responses. An accurate description of the nuclear quasi-elastic responses requires the inclusion of effects beyond those considered by the RPA. These calculations are unfortunately rather involved, especially for finite nuclear systems. Probably they can be affordable in infinite nuclear matter and in this case our smoothed Fermi surface prescription could be extremely useful for reliable results. Furthermore, its use is computationally much less demanding than the common LDA.

\section{Acknowledgments}

We thank A M Lallena for useful discussions. This work has been partially supported by MURST through the Progetto di Ricerca di Interesse Nazionale: Fisica teorica del nucleo atomico e dei sistemi a molticorpi.

\section{References}

[1] Cavinato M, Drechsel D, Fein E, Marangoni M and Saruis A M 1984 Nucl. Phys. A 423376 Cavinato M, Marangoni M and Saruis A M 1990 Phys. Lett. B 235346 Saruis A M 1993 Phys. Rep. 23557

[2] Dellafiore A, Lenz F and Brieva F A 1985 Phys. Rev. C 311088 Brieva F A and Dellafiore A 1987 Phys. Rev. C 36899

[3] Shigehara T, Shimizu K and Arima A 1989 Nucl. Phys. A 492388

[4] Buballa M, Drożdż S, Krewald S and Speth J 1991 Ann. Phys., NY 208346 Buballa M, Drożdż S, Krewald S and Szczurek S 1991 Phys. Rev. C 44810 Jeschonnek S, Szczurek S, Co' G and Krewald S 1994 Nucl. Phys. A 570599

[5] Co' G, Quader K F, Smith D R and Wambach J 1988 Nucl. Phys. A 48561

[6] Drożdż S, Co’ G, Wambach J and Speth J 1987 Phys. Lett. B 185287 Drożdż S, Nishizaki S, Speth J and Wambach J 1990 Phys. Rep. 1971

[7] Takayanagi K, Shimizu K and Arima A 1988 Nucl. Phys. A 477205 Takayanagi K 1989 Phys. Lett. B 23011

Takayanagi K 1990 Nucl. Phys. A 510162 Takayanagi K 1991 Nucl. Phys. A 522494 Takayanagi K 1991 Nucl. Phys. A 522523 Takayanagi K 1993 Nucl. Phys. A 55614

[8] Chinn C R, Picklesimer A and Van Orden J W 1989 Phys. Rev. C 401159 Capuzzi F, Giusti C and Pacati F D 1991 Nucl. Phys. A 524681 Capuzzi F 1993 Nucl. Phys. A 554362

[9] Amaro J E, Co' G and Lallena A M 1993 Ann. Phys., NY 221306 Amaro J E, Co' G and Lallena A M 1994 Nucl. Phys. A 578365

[10] Co' G and Lallena A M 2001 Ann. Phys., NY 287101

[11] Amaro J E, Lallena A M and Co' G 1994 Int. J. Mod. Phys. E 3735

[12] Alberico W M, Barbaro M B, de Pace A, Donnelly T W and Molinari A 1993 Nucl. Phys. A 563605 Barbaro M B, de Pace A, Donnelly T W and Molinari A 1996 Nucl. Phys. A 596553 
Barbaro M B, de Pace A, Donnelly T W and Molinari A 1996 Nucl. Phys. A 598503

de Pace A 1998 Nucl. Phys. A 635163

[13] Bauer E, Ramos A and Polls A 1996 Phys. Rev. C 542959

[14] Bauer E and Lallena A M 1999 Phys. Rev. C 592603

[15] Bauer E 2000 J. Phys. G: Nucl. Part. Phys. 26145

[16] Alberico W M, Ericson M and Molinari A 1984 Ann. Phys., NY 154356

Alberico W M, de Pace A, Drago A and Molinari A 1991 Riv. Nuovo Cimento 141

Alberico W M, Donnelly T W and Molinari A 1990 Nucl. Phys. A 512541

[17] Bauer E 1995 Nucl. Phys. A 589669

Bauer E, Polls A and Ramos A 1998 Phys. Rev. C 581052

[18] Fantoni S and Pandharipande V R 1984 Nucl. Phys. A 427473

Fabrocini A and Fantoni S 1989 Nucl. Phys. A 503375

Benhar O, Fabrocini A and Fantoni S 1992 Nucl. Phys. A 550201

[19] Gil A, Nieves J and Oset E 1997 Nucl. Phys. A 627543

Gil A 1996 PhD Thesis Universitat de Valencia (unpublished)

[20] Cenni R, Conte F and Saracco P 1997 Nucl. Phys. A 623391

[21] Bauer E 1998 Nucl. Phys. A 637243

[22] Amaro J E, Barbaro M B, Caballero J A, Donnelly T W and Molinari A 1999 Nucl. Phys. A 657161

[23] Van Orden J W and Donnelly T W 1981 Ann. Phys., NY 131451

[24] de Haro R, Krewald S and Speth J 1982 Nucl. Phys. A 388265

Co' G and Krewald S 1985 Nucl. Phys. A 433392

[25] Bauer E 2000 Phys. Rev. C 61044307

[26] Rinker G and Speth J 1978 Nucl. Phys. A 306360

[27] Barreau P et al 1983 Nucl. Phys. A 402515

[28] Williamson C F et al 1997 Phys. Rev. C 563152

[29] Pines D, Quader K F and Wambach J 1988 Nucl. Phys. A 477365 